\title{
Dysphagia Hinders Hospitalized Patients with Heart Failure from Being Discharged to Home
}

\author{
Junichi Yokota, ${ }^{1,2}$ Yoshiko Ogawa, ${ }^{3}$ Yoshimi Takahashi, ${ }^{4}$ Nobuhiro Yamaguchi, ${ }^{4}$ \\ Noriko Onoue, ${ }^{4}$ Tsuyoshi Shinozaki ${ }^{4}$ and Masahiro Kohzuki ${ }^{1}$ \\ ${ }^{1}$ Department of Internal Medicine and Rehabilitation Science Disability Science, Tohoku University Graduate \\ School of Medicine, Sendai, Miyagi, Japan \\ ${ }^{2}$ Department of Rehabilitation, National Hospital Organization Sendai Medical Center, Sendai, Miyagi, Japan \\ ${ }^{3}$ Department of Sport and Medical Sciences, Teikyo University, Hachioji, Tokyo, Japan \\ ${ }^{4}$ Department of Cardiology, National Hospital Organization Sendai Medical Center, Sendai, Miyagi, Japan
}

\begin{abstract}
Dysphagia, defined as a dysfunction in any stage or process of eating, is common among heart failure (HF) patients. In some diseases state, dysphagia hinders patients from being discharged to home. However, it remains unclear whether dysphagia affects discharge disposition of HF patients. This study aimed to identify the impact of dysphagia on discharge disposition of HF patients. A total of 323 patients, hospitalized with acute exacerbation of HF, were eligible for the study (excluding patients who lived at nursing care facilities before admission). Following the withdrawal of 37 patients, a total of 286 patients were analyzed. Dysphagia was determined using the functional oral intake scale (FOIS), which evaluates a patient's ability to swallow. The FOIS is a 7-point scale, with a level of $\leq 5$ indicating dysphagia. Of the 286 patients analyzed, 231 (80.8\%) were discharged to home, and 55 were discharged to nursing care facilities or rehabilitation hospitals (non-home). FOIS level was significantly lower, and dysphagia incidence was significantly higher among patients discharged to non-home than among those discharged to home. Multivariate analysis showed that FOIS level was an independent predictor of discharge disposition. Additionally, after propensity score matching, which was performed to adjust for baseline characteristics, FOIS level remained significantly lower in patients discharged to non-home than in those discharged to home. In conclusion, dysphagia hinders patients hospitalized with HF from being discharged to home. We conclude that evaluating dysphagia and its severity on admission is useful for predicting discharge disposition in patients hospitalized with HF.
\end{abstract}

Keywords: discharge disposition; dysphagia; functional oral intake scale; heart failure; swallowing function Tohoku J. Exp. Med., 2019 November, 249 (3), 163-171. (C) 2019 Tohoku University Medical Press

\section{Introduction}

Heart failure (HF) is a global problem and is defined by the inability of the heart to pump sufficient blood to the tissues of the body. In Japan, the number of HF patients is increasing due to the aging population (Shimokawa et al. 2015). Some elderly patients hospitalized with cardiovascular disorders, such as post-cardiac surgery, acute myocardial infarction, and peripheral artery disease, cannot be discharged to their homes and are forced to be admitted to nursing care facilities or rehabilitation hospitals (Sansone et al. 2002; Sasanuma et al. 2015). Previous studies have reported that advanced age, lower body mass index (BMI), a higher number of comorbidities, and lower levels of activities of daily living (ADL) prevent patients with cardiovascular disorders from being discharged to home (Sansone et al. 2002; Sasanuma et al. 2015).
In the broad sense, the term "dysphagia" refers to the dysfunction in any stage or process of eating (the pre-oral, oral, pharyngeal, and esophageal phase) (Clark et al. 2007). Eating is a complex activity that requires effective and coordinated function of the motor, sensory, and cognitive systems. Therefore, dysphagia can be caused by neurological, muscular, and cognitive impairment concomitant with various disease states, aging, surgical intervention, intubation, and other conditions. Elderly people often have various physical and cognitive impairments associated with the aging process, and dysphagia is a common and significant problem among this demographic group (Hansen et al. 2011).

Patients with dysphagia can be screened for using a questionnaire (Belafsky et al. 2008), a repetitive saliva swallowing test (RSST) (Oguchi et al. 2000a, b) and a water swallowing test (WST) (Tohara et al. 2003) and can

Received July 22, 2019; revised and accepted October 23, 2019. Published online November 14, 2019; doi: 10.1620/tjem.249.163.

Correspondence: Masahiro Kohzuki, M.D., Ph.D., Department of Internal Medicine and Rehabilitation Science Disability Science,

Tohoku University Graduate School of Medicine, 1-1 Seiryo-machi, Aoba-ku, Sendai, Miyagi 980-8574, Japan.

e-mail: kohzuki@med.tohoku.ac.jp 
be diagnosed using videofluoroscopic and videoendoscopic evaluation of swallowing. However, depending on their clinical condition, not all patients are able to take these examinations. The functional oral intake scale (FOIS) is an evaluation method for swallowing (Crary et al. 2005). The FOIS is a 7-point scale that measures the level of independent functional oral intake (Table 1). Since the use of FOIS is non-invasive, it can be used in all patients regardless of their clinical condition. Moreover, the FOIS can evaluate the severity of dysphagia, with clinically significant dysphagia being defined as the requirement for modification of an oral diet (FOIS level $\leq 5$ ) (Maeda and Akagi 2015).

Dysphagia is a common comorbidity in patients with $\mathrm{HF}$, with $36.1 \%$ of patients hospitalized with acute exacerbation of HF also showing symptoms of dysphagia (Yokota et al. 2016). Previous studies have demonstrated that dysphagia hinders patients hospitalized with certain diseases from being discharged to home. In acute stroke patients with dysphagia, compared with patients without dysphagia, the percentage of patients who were not discharged to home was increased by $36 \%$ (Joundi et al. 2017). Moreover, in survivors of critical illness and critical illness with neurologic impairment (Macht et al. 2011, 2013), the presence of post-extubation dysphagia has been found to increase the percentage of patients who are not discharged to home (by $29 \%$ and $14 \%$, respectively) in comparison with patients without post-extubation dysphagia. However, it remains unclear whether dysphagia affects discharge disposition in HF patients. This study aimed to identify the impact of dysphagia on the discharge disposition of hospitalized patients with HF.

\section{Materials and Methods}

\section{Subjects and Study Design}

This study used a longitudinal design, as shown in the study protocol (Fig. 1). In total, 412 patients were hospitalized at the Department of Cardiology with acute exacerbation of HF from May 2015 to October 2018. HF was diagnosed by cardiologists based on the guidelines of the Japan Circulation Society (Tsutsui 2017). Patients excluded were those aged $<20$ years $(n=0)$, those who lived in nursing care facilities before admission $(n=19)$, and those unable to follow instructions due to severe mental disorders, severe dementia, or agitation $(n=20)$. A further 50 patients did not provide consent to participate, leaving a total of 323 patients eligible for enroll- ment in the study. After registration, the baseline characteristics of the patients were collected. Swallowing function was evaluated when the attending physicians allowed patients to eat and drink. The discharge disposition (the final place to which the patient was discharged) was recorded on the day of discharge. During the follow-up period, 37 patients were withdrawn due to death, transfer to other departments, cardiovascular surgery, or stroke. This led to a final analysis of 286 patients.

This study was approved by the Ethics Committee of Tohoku University Graduate School of Medicine (Approval No. 2015-1-060) and the Ethics Committee of National Hospital Organization Sendai Medical Center (Approval No. 27-1). All participating patients provided written informed consent.

\section{Patient Characteristics on admission}

Clinical data, including age, sex, height, weight, BMI, medical history, complications, medications, the number of people living together, New York Heart Association (NYHA) class, ejection fraction (EF), and blood chemistry data were obtained on admission. NYHA class, an index of heart failure severity, was diagnosed by cardiologists.

Cognitive function was evaluated using the mini-mental state examination (MMSE) (Folstein et al. 1975). Physical function was evaluated by handgrip strength (Izawa et al. 2004), the modified specific activity scale (MSAS; modified from the specific activity scale (Goldman et al. 1981) to improve sensitivity and specificity) (Adachi et al. 2009), and the Barthel index (BI) (an evaluation scale of ADL) (Mahoney and Barthel 1965). Controlling nutritional status (CONUT) (Ignacio de Ulibarri et al. 2005), arm circumference (AC), and triceps skin fold (TSF) (Hosoya et al. 2001) were measured to evaluate nutritional status. CONUT is a nutritional assessment tool that assesses protein metabolism, lipid metabolism, and immune function and is calculated using three parameters: serum albumin, total cholesterol, and total lymphocyte count. CONUT consists of a 12-point scale; a score of 0-1 indicates normal nutrition, 2-4 indicates mild malnutrition, 5-8 indicates moderate malnutrition, and $>7$ indicates severe malnutrition. AC and TSF data were standardized according to age and sex-stratified Japanese anthropometric reference data and expressed as percentage AC (\%AC) and percentage TSF (\%TSF) (Hosoya et al. 2001). Cognitive function, physical function, and nutritional status were evaluated by physiotherapists and occupational therapists.

\section{Swallowing Function}

Dysphagia was evaluated using FOIS. Based on a previous study, dysphagia was defined as a FOIS level $\leq 5$ (Maeda and Akagi

Table 1. Functional Oral Intake Scale.

\begin{tabular}{ll}
\hline Level 1 & Nothing by mouth. \\
Level 2 & Tube dependent with minimal attempts of food or liquid. \\
Level 3 & Tube dependent with consistent oral intake of food or liquid. \\
Level 4 & Total oral diet of a single consistency. \\
Level 5 & $\begin{array}{l}\text { Total oral diet with multiple consistencies, } \\
\text { but requiring special preparation or compensations. }\end{array}$ \\
Level 6 & $\begin{array}{l}\text { Total oral diet with multiple consistencies without special preparation, } \\
\text { but with specific food limitations. }\end{array}$ \\
Level 7 & Total oral diet with no restrictions. \\
\hline
\end{tabular}




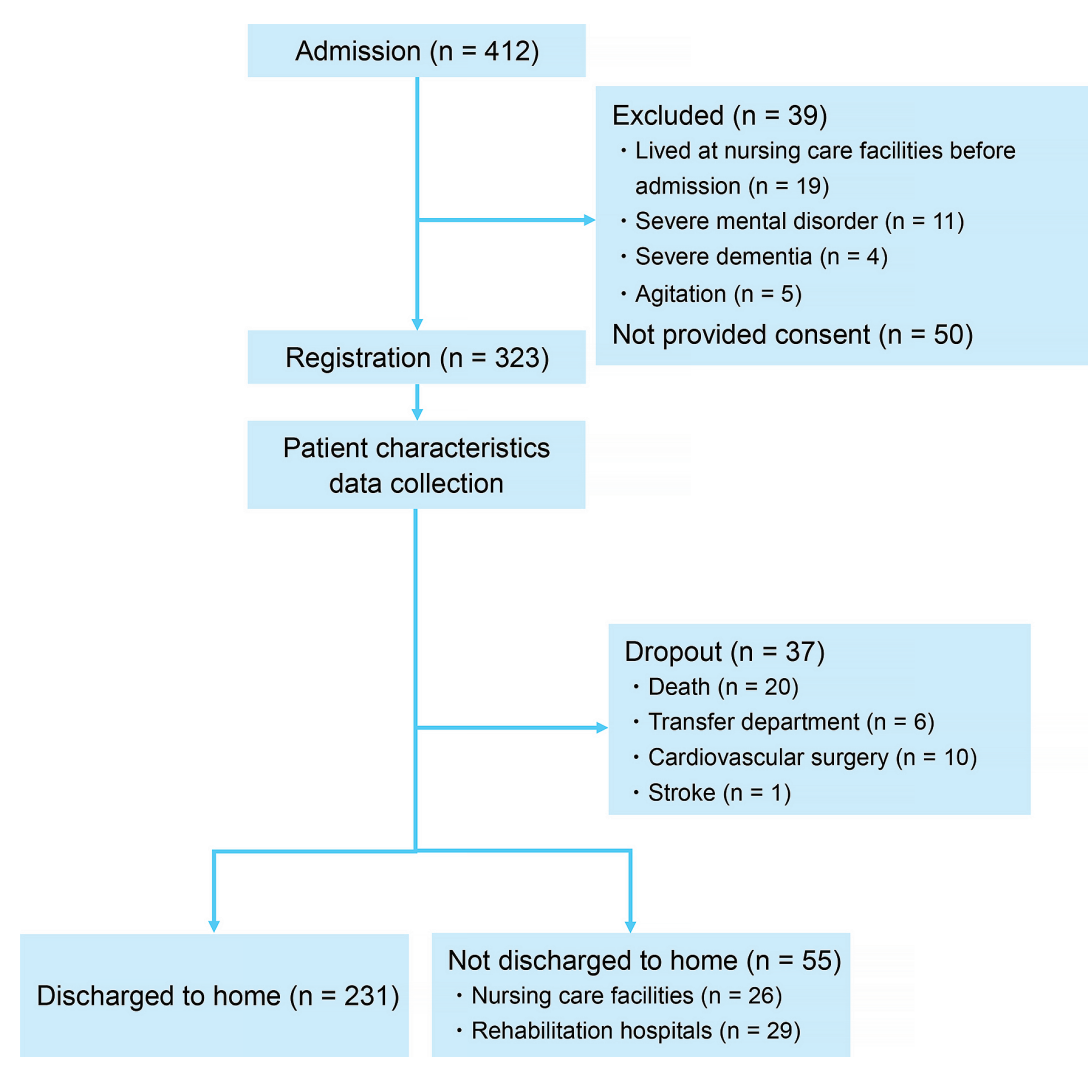

Fig. 1. Study protocol.

A total of 412 patients were admitted to the Department of Cardiology with acute exacerbation of heart failure. Those excluded from the study were patients aged $<20$ years, those living in nursing care facilities before admission, and those unable to follow instructions. A further 50 patients did not provide consent to participate. Thus, 323 patients were eligible for the study. After registration, the baseline characteristics of these patients were collected. Swallowing function was evaluated when the attending physicians allowed patients to eat and drink. The discharge disposition was recorded at discharge. Due to the withdrawal of 37 patients, 286 patients were analyzed.

2015). Additionally, swallowing function was evaluated using RSST and WST. These were performed to assess general pharyngeal function in swallowing. RSST assesses the ability to swallow saliva. The test was performed by counting the frequency of swallowing over 30 seconds. The swallowing function of the patients was judged as abnormal when the number of dry swallows was less than 3 within 30 seconds. The WST assessed the ability to swallow water. The WST was performed by placing cold water $(3 \mathrm{ml})$ on the floor of the mouth and instructing the patient to swallow. If the patient was unable to swallow, or experienced dyspnea, coughing, or wet-hoarse dysphonia after swallowing, the test was concluded and a score was judged (1 for inability to swallow, 2 for dyspnea, and 3 for cough or dysphonia). Otherwise, the patient was asked to perform two dry swallows. If the patient was unable to perform either of these, a score of 4 was assigned. If the patient was able to complete the dry swallowing challenge, a score of 5 was assigned. The entire procedure was repeated a total of three times. The swallowing function of the patient was determined to be abnormal when the score was less than 4 . Swallowing function was evaluated by physicians, nurses, and speech-language-hearing therapists.

\section{Rehabilitation Intervention}

The number of patients who had undergone physical therapy or swallowing therapy during hospitalization was checked using electronic medical records.

\section{Data and Statistical Analysis}

Patients were divided into two groups based on their discharge disposition: those discharged to home (home group), and those discharged to nursing care facilities or rehabilitation hospitals (non-home group). All data are expressed as the mean \pm standard deviation for continuous variables, and as counts and percentages for categorical variables. Comparisons between groups were made using an unpaired two-tailed $t$-test for continuous variables and the chi-square test for categorical variables. The independent predictors of discharge to home were assessed using logistic regression analysis. Variables that showed significant differences between groups in the univariate analysis were entered into a logistic regression model, and multicollinearity was assessed using the variance inflation factor (VIF) (Glantz and Slinker 2001). VIF values exceeding 10 were considered indicative of serious multicollinearity (Glantz and Slinker 2001). Predictors were expressed as odds ratios with $95 \%$ confidence intervals. Moreover, the cut-off values were assessed using a receiver operating characteristic (ROC) curve.

Additionally, to adjust for significant differences in baseline characteristics between the home and non-home groups, we conducted propensity score matching by a logistic regression model based on the following variables: age, sex, height, weight, NYHA class, EF, blood chemistry parameters (excluding albumin and transthyretin), MMSE, handgrip strength, MSAS, BI, \%AC, and \%TSF. The discrimination capability of this model was evaluated using 
c-statistics $(0.887)$. The propensity score matching was performed in a 1 to 1 manner, with a caliper width of 0.25 . The clinical characteristics of the two matched groups were compared.

All analyses were performed using JMP version 13.1.0 (SAS Institute, Cary, NC, USA). Statistical significance was determined as a $\mathrm{P}$ value of $<0.05$.

\section{Results}

\section{Patient Characteristics on admission}

Among the 286 patients, 231 (80.8\%) were discharged to home (home group) and 55 were discharged to nursing care facilities or rehabilitation hospitals (non-home group). Table 2 shows the patient characteristics on admission in both groups. Age and percentage of females were significantly higher while height, weight, and BMI were significantly lower in the non-home group than in the home group. The percentage of patients with a medical history of dementia was significantly higher in the non-home group than in the home group. Albumin level was significantly lower in the non-home group than in the home group. There were no significant differences in the number of people living together, NYHA class, EF, and blood chemistry data (excluding albumin) between the two groups. In this study, 111 patients $(38.8 \%)$ were diagnosed with HF with preserved EF $(\geq 50 \%)$, of which 88 patients were in the home group (38.1\%) and 23 were in the non-home group $(41.8 \%)$.

There were no significant differences between the groups in the days from hospitalization to evaluation in terms of cognitive function, physical function, and nutritional status (home group, $3.5 \pm 2.6$ days vs non-home group, $4.1 \pm 3.4$ days, $\mathrm{P}=0.232$ ). All indexes of cognitive function, physical function, and nutritional status (excluding CONUT) were significantly lower in the non-home group than in the home group.

\section{Swallowing Function}

There was no significant difference in the number of days between hospital admission and swallowing assessment between the two groups (home group, $2.7 \pm 1.9$ days vs non-home group, $3.0 \pm 2.4$ days, $\mathrm{P}=0.403$ ). The FOIS level was significantly lower and the percentage of patients with FOIS level $\leq 5$ was significantly higher in the nonhome group than in the home group. The percentage of patients with a positive RSST was significantly higher, and the WST score was significantly lower in the non-home group than in the home group (Table 2).

\section{Outcomes and Rehabilitation Intervention}

Length of hospital stay was significantly longer in the non-home group than in the home group. The percentage of patients provided with physical therapy or swallowing therapy interventions was significantly higher in the nonhome group than in the home group (Table 3).

\section{Predictors of discharge to home}

Table 4 shows the predictors for discharge to home. Variables that showed significant differences between groups in the univariate analysis were entered, and there were no variables that had VIF values that exceeded 10 . Significant differences were seen in FOIS level, handgrip strength, and \%TSF. Additionally, the cut-off values for predicting discharge to home were evaluated using a ROC curve. Values are shown in Table 5.

\section{Comparison between the groups after propensity score matching}

After propensity score matching was performed, FOIS level remained significantly lower, and the percentage of patients with FOIS level $\leq 5$ remained significantly higher in the non-home group than in the home group. The percentage of patients with a positive RSST was significantly higher, and WST scores were significantly lower in the nonhome group than in the home group. Length of hospital stay was significantly prolonged in the non-home group in comparison with the home group. There were no significant differences in the other parameters between the groups (Tables 6 and 7).

\section{Discussion}

Dysphagia is a common comorbidity among heart failure patients and is believed to play a role in hindering patients from being discharged to home. This study aimed to identify the impact of dysphagia on the discharge disposition of patients with HF

In the current study, the percentage of patients with dysphagia was higher in hospitalized HF patients who were discharged to nursing care facilities or rehabilitation hospitals than those who were discharged to home. Moreover, this study clarified that FOIS level was an independent predictor of discharge to home in patients hospitalized with acute exacerbation of HF, with a FOIS level $>5$ indicating a high chance of being discharged to home. In a previous study, dysphagia was defined as having a FOIS level $\leq 5$ (Maeda and Akagi 2015). Therefore, this study demonstrates the correlation between dysphagia and discharge disposition and indicates that dysphagia hinders patients hospitalized with HF from being discharged to home.

Based on multivariate analysis, handgrip strength and \%TSF were also independent predictors of discharge to home in patients hospitalized with acute exacerbation of HF. However, after propensity score matching, which was performed to adjust for the baseline characteristics that can affect discharge disposition in HF, handgrip strength and \%TSF were not significantly different between HF patients of either discharge disposition. On the other hand, FOIS level was significantly lower in HF patients who discharged to nursing care facilities or rehabilitation hospitals, and the percentage of patients with a FOIS level $\leq 5$ remained significantly higher after propensity score matching. Additionally, the results of the evaluation of swallowing 
Table 2. Patient characteristics on admission.

\begin{tabular}{|c|c|c|c|}
\hline & Home $(n=231)$ & Non-home $(n=55)$ & $P$ value \\
\hline \multicolumn{4}{|l|}{ Basic characteristics } \\
\hline Age (years) & $79.3 \pm 10.1$ & $85.1 \pm 9.4$ & $<0.001$ \\
\hline Sex (male/female) & $109 / 122$ & $15 / 40$ & 0.010 \\
\hline Height $(\mathrm{cm})$ & $155.6 \pm 10.1$ & $151.3 \pm 10.0$ & 0.004 \\
\hline Weight (kg) & $58.8 \pm 12.8$ & $49.9 \pm 11.8$ & $<0.001$ \\
\hline BMI $\left(\mathrm{kg} / \mathrm{m}^{2}\right)$ & $24.2 \pm 4.4$ & $21.8 \pm 4.4$ & $<0.001$ \\
\hline \multicolumn{4}{|l|}{ Medical history } \\
\hline Angina pectoris & $60(26.0)$ & $9(16.4)$ & 0.162 \\
\hline Myocardial infarction & $32(13.9)$ & $8(14.5)$ & 0.894 \\
\hline Valvular disease & $52(22.5)$ & $12(21.8)$ & 0.912 \\
\hline Cerebrovascular disease & $52(22.5)$ & $11(20.0)$ & 0.686 \\
\hline Neuromuscular disease & $4(1.7)$ & $1(1.8)$ & 0.965 \\
\hline Respiratory disease & $21(9.1)$ & $3(5.5)$ & 0.382 \\
\hline Cancer & $44(19.0)$ & $8(14.5)$ & 0.437 \\
\hline Dementia & $17(7.4)$ & $13(23.6)$ & $<0.001$ \\
\hline \multicolumn{4}{|l|}{ Complication } \\
\hline Diabetes & $103(44.6)$ & $22(40.0)$ & 0.538 \\
\hline Hypertension & $175(75.8)$ & $37(67.3)$ & 0.197 \\
\hline Dyslipidemia & $71(30.7)$ & $15(27.3)$ & 0.615 \\
\hline \multicolumn{4}{|l|}{ Medication } \\
\hline ACEI & $61(26.4)$ & $10(18.2)$ & 0.204 \\
\hline ARB & $96(41.6)$ & $22(40.0)$ & 0.833 \\
\hline Statin & $55(23.8)$ & $10(18.2)$ & 0.106 \\
\hline Calcium antagonists & $89(38.5)$ & $18(32.7)$ & 0.424 \\
\hline Diuretics & $215(93.1)$ & $50(90.9)$ & 0.775 \\
\hline Beta-blockers & $77(33.3)$ & $12(21.8)$ & 0.097 \\
\hline Coronary vasodilators & $55(28.2)$ & $16(28.1)$ & 0.797 \\
\hline Psychotropic drug & $13(5.6)$ & $6(10.9)$ & 0.222 \\
\hline Anti-dementia drug & $14(6.1)$ & $2(3.6)$ & 0.482 \\
\hline Number of people living together & $1.5 \pm 1.2$ & $1.3 \pm 1.6$ & 0.473 \\
\hline \multicolumn{4}{|l|}{ Clinical and laboratory findings } \\
\hline NYHA class (I /II / III / IV) & $7 / 41$ / 137 / 46 & $0 / 5 / 35 / 15$ & 0.172 \\
\hline Ejection fraction (\%) & $50.9 \pm 17.5$ & $53.3 \pm 17.8$ & 0.368 \\
\hline NT-proBNP (pg/mL) & $9,688.7 \pm 13,154.7$ & $12,591.8 \pm 12,615.2$ & 0.142 \\
\hline Hemoglobin $(\mathrm{g} / \mathrm{dL})$ & $11.3 \pm 2.4$ & $10.8 \pm 1.8$ & 0.102 \\
\hline Total lymphocyte count $(/ \mu \mathrm{L})$ & $1,367.7 \pm 759.7$ & $1,239.8 \pm 758.1$ & 0.263 \\
\hline eGFR $\left(\mathrm{mL} / \mathrm{min} / 1.73 \mathrm{~m}^{2}\right)$ & $48.3 \pm 25.3$ & $41.2 \pm 27.9$ & 0.068 \\
\hline CRP (mg/dL) & $1.8 \pm 3.1$ & $2.6 \pm 4.1$ & 0.162 \\
\hline Albumin (g/dL) & $3.5 \pm 0.5$ & $3.4 \pm 0.5$ & 0.032 \\
\hline Transthyretin (mg/dL) & $17.0 \pm 5.5$ & $16.3 \pm 5.6$ & 0.350 \\
\hline Total cholesterol (g/dL) & $162.0 \pm 40.8$ & $167.1 \pm 34.6$ & 0.386 \\
\hline \multicolumn{4}{|c|}{ Cognitive function, physical function, and nutritional status } \\
\hline MMSE (score) & $23.1 \pm 4.9$ & $17.9 \pm 6.7$ & $<0.001$ \\
\hline Handgrip strength (kg) & $20.2 \pm 7.7$ & $12.8 \pm 6.3$ & $<0.001$ \\
\hline MSAS (METs) & $2.2 \pm 1.0$ & $1.9 \pm 0.5$ & 0.001 \\
\hline $\mathrm{BI}$ (score) & $56.6 \pm 28.5$ & $32.1 \pm 24.6$ & $<0.001$ \\
\hline CONUT (score) & $3.6 \pm 2.4$ & $3.9 \pm 2.3$ & 0.479 \\
\hline$\% A C(\%)$ & $104.1 \pm 18.9$ & $96.0 \pm 15.0$ & 0.003 \\
\hline$\%$ TSF $(\%)$ & $96.2 \pm 49.6$ & $70.1 \pm 40.6$ & $<0.001$ \\
\hline \multicolumn{4}{|l|}{ Swallowing function } \\
\hline FOIS (level) & $6.1 \pm 1.5$ & $4.3 \pm 2.0$ & $<0.001$ \\
\hline Number of patients with FOIS level $\leq 5$ & $51(22.1)$ & $34(61.8)$ & $<0.001$ \\
\hline RSST positive & $51(22.1)$ & $32(58.2)$ & $<0.001$ \\
\hline WST (score) & $4.4 \pm 0.9$ & $3.7 \pm 0.9$ & $<0.001$ \\
\hline
\end{tabular}

Values are means \pm standard deviation or numbers of subjects per group (n) with percentages.

$\mathrm{AC}$, arm circumference; ACEI, angiotensin converting enzyme inhibitor; ARB, angiotensin receptor blocker; BI, barthel index; BMI, body mass index; CONUT, controlling nutritional status; CRP, C-reactive protein; FOIS, functional oral intake scale; eGFR, estimated glomerular filtration rate; METs, metabolic equivalents; MMSE, mini-mental state examination; MSAS, modified specific activity scale; NT-proBNP, N-terminal pro-B-type natriuretic peptide; NYHA, New York Heart Association; RSST, repetitive saliva swallowing test; TSF, triceps skin fold; WST, water swallowing test. 
Table 3. Outcomes and Rehabilitation Interventions.

\begin{tabular}{lccr}
\hline & Home $(\mathrm{n}=231)$ & Non-home $(\mathrm{n}=55)$ & P value \\
\hline Outcomes & & & \\
Length of hospital stay (day) & $28.9 \pm 15.2$ & $45.4 \pm 34.7$ & 0.001 \\
$\begin{array}{l}\text { Discharge disposition } \\
\text { Home }\end{array}$ & $231(100.0)$ & $0(0.0)$ & $<0.001$ \\
$\quad$ Nursing care facility & $0(0.0)$ & $26(47.3)$ & \\
$\quad$ Rehabilitation hospital & $0(0.0)$ & $29(62.7)$ & \\
Rehabilitation interventions & $188(81.4)$ & $52(94.5)$ & 0.017 \\
PT & $9(3.9)$ & $16(29.1)$ & $<0.001$ \\
ST & &
\end{tabular}

Table 4. Predictors of discharge to home.

\begin{tabular}{lcccccc}
\hline & $\beta$ & SE & Wald & P-value & OR & $95 \% \mathrm{Cl}$ \\
\hline FOIS (level) & -0.422 & 0.096 & 19.534 & $<0.001$ & 0.655 & $-0.610--0.235$ \\
Handgrip strength (kg) & -0.031 & 0.031 & 22.378 & 0.001 & 0.863 & $-0.208-0.086$ \\
\%TSF & -0.014 & 0.004 & 10.654 & $<0.001$ & 0.986 & $-0.022--0.006$ \\
\hline
\end{tabular}

CI, confidence interval; FOIS, functional intake oral scale; OR, odds ratio; SE, standard error; TSF, triceps skin fold.

Table 5. Cut-off values of discharge to home.

\begin{tabular}{lccccc}
\hline & AUC & P-value & Cut-off & Sensitivity & Specificity \\
\hline FOIS (level) & 0.749 & $<0.001$ & $>5$ level & $81.4 \%$ & $60.0 \%$ \\
Handgrip strength (kg) & 0.783 & $<0.001$ & $>12.6 \mathrm{~kg}$ & $87.0 \%$ & $61.8 \%$ \\
$\%$ TSF & 0.657 & $<0.001$ & $>85.7 \%$ & $52.8 \%$ & $76.4 \%$ \\
\hline
\end{tabular}

AUC, area under curve; CI, confidence interval; FOIS, functional intake oral scale; TSF, triceps skin fold.

function using RSST and WST were lower in HF patients who discharged to nursing care facilities or rehabilitation hospitals after propensity score matching. These results suggest that dysphagia itself hinders patients hospitalized with acute exacerbation of HF from being discharged to home. Furthermore, it was demonstrated that the severity of dysphagia positively correlated with the risk of discharge to nursing care facilities or rehabilitation hospitals in patients with acute exacerbation of HF. These new findings indicate that a FOIS level can be used for predicting discharge disposition and may be valuable in identifying patients who need special interventions.

The mechanisms by which dysphagia negatively affects discharge disposition remain unclear. However, previous studies have reported that in certain disease states, dysphagia increases the percentage of patients who cannot be discharged to home after hospitalization (Macht et al. 2011, 2013; Joundi et al. 2017). In the context of this study, dysphagia may cause aggravation of clinical outcomes in patients with acute exacerbation of HF, through poor ADL as a result of malnutrition and muscle wasting. Indeed, studies have shown that dysphagia causes malnutrition and muscle wasting in elderly patients in various disease states (Serra-Prat et al. 2012; Morley 2018). Malnutrition and muscle wasting results in poor ADL, thereby preventing patients with certain disease states from being discharged to home (Sansone et al. 2002; Sasanuma et al. 2015; Isono et al. 2017).

In this study, the percentage of patients with dysphagia provided with physical therapy was relatively high, but the percentage of patients provided with swallowing therapy was relatively low. More active intervention to improve swallowing function may decrease the percentage of patients with poor ADL and increase the percentage of patients with exacerbation of HF that discharge to home.

This study has several limitations. Firstly, this study was conducted at a single center, and may not reflect the findings of all other centers. Secondly, the state of patients at discharge, including severity of dysphagia, was not examined. Swallowing function may have changed during hospitalization, and changes in swallowing function may have contributed to discharge disposition. Thirdly, the total time and content of cardiac rehabilitation during hospitalization were unclear. Therefore, further studies such as 
Table 6. Patient characteristics on admission after propensity score matching.

\begin{tabular}{|c|c|c|c|}
\hline & Home $(n=41)$ & Non-home $(n=41)$ & $P$ value \\
\hline \multicolumn{4}{|l|}{ Basic characteristics } \\
\hline Age (years) & $82.3 \pm 7.3$ & $84.2 \pm 10.0$ & 0.451 \\
\hline Sex (male/female) & $11 / 30$ & $13 / 28$ & 0.627 \\
\hline Height $(\mathrm{cm})$ & $151.4 \pm 9.2$ & $151.7 \pm 10.9$ & 0.879 \\
\hline Weight (kg) & $52.1 \pm 9.8$ & $50.1 \pm 12.8$ & 0.667 \\
\hline $\operatorname{BMI}\left(\mathrm{kg} / \mathrm{m}^{2}\right)$ & $22.8 \pm 3.7$ & $21.8 \pm 4.4$ & 0.450 \\
\hline \multicolumn{4}{|l|}{ Medical history } \\
\hline Angina pectoris & $7(17.1)$ & $8(19.5)$ & 0.775 \\
\hline Myocardial infarction & $6(14.6)$ & $4(9.8)$ & 0.500 \\
\hline Valvular disease & $9(22.0)$ & $10(24.4)$ & 0.794 \\
\hline Cerebrovascular disease & $4(9.8)$ & $8(19.5)$ & 0.211 \\
\hline Neuromuscular disease & $0(0.0)$ & $1(2.4)$ & 0.314 \\
\hline Respiratory disease & $1(2.4)$ & $3(7.3)$ & 0.305 \\
\hline Cancer & $10(24.4)$ & $6(14.6)$ & 0.265 \\
\hline Dementia & $8(19.5)$ & $8(19.5)$ & 1.000 \\
\hline \multicolumn{4}{|l|}{ Complication } \\
\hline Diabetes & $17(41.5)$ & $16(39.0)$ & 0.821 \\
\hline Hypertension & $32(78.1)$ & $27(65.9)$ & 0.219 \\
\hline Dyslipidemia & $10(24.4)$ & $12(29.3)$ & 0.618 \\
\hline \multicolumn{4}{|l|}{ Medication } \\
\hline ACEI & $11(26.8)$ & $7(17.1)$ & 0.286 \\
\hline ARB & $15(36.6)$ & $18(43.9)$ & 0.499 \\
\hline Statin & $4(9.8)$ & $7(17.1)$ & 0.331 \\
\hline Calcium antagonists & $16(39.0)$ & $14(34.2)$ & 0.424 \\
\hline Diuretics & 39 (95.1) & $37(90.2)$ & 0.396 \\
\hline Beta-blockers & $11(26.8)$ & $11(26.8)$ & 1.000 \\
\hline Coronary vasodilators & $10(24.4)$ & $12(29.3)$ & 0.618 \\
\hline Psychotropic drug & $2(4.9)$ & $5(12.2)$ & 0.236 \\
\hline Anti-dementia drug & $3(7.3)$ & $0(0.0)$ & 0.078 \\
\hline Number of people living together & $1.8 \pm 1.3$ & $1.5 \pm 1.5$ & 0.330 \\
\hline \multicolumn{4}{|l|}{ Clinical and laboratory findings } \\
\hline NYHA class (I /II / III / IV) & $0 / 3 / 28 / 10$ & $0 / 4 / 28 / 9$ & 0.901 \\
\hline Ejection fraction $(\%)$ & $50.1 \pm 19.0$ & $54.1 \pm 18.4$ & 0.344 \\
\hline NT-proBNP (pg/mL) & $13,876.8 \pm 16,470.7$ & $13,064.5 \pm 12,916.9$ & 0.804 \\
\hline Hemoglobin $(\mathrm{g} / \mathrm{dL})$ & $10.5 \pm 2.2$ & $11.0 \pm 1.6$ & 0.227 \\
\hline Total lymphocyte count $(/ \mu \mathrm{L})$ & $1,233.9 \pm 650.3$ & $1,336.1 \pm 822.0$ & 0.534 \\
\hline eGFR $\left(\mathrm{mL} / \mathrm{min} / 1.73 \mathrm{~m}^{2}\right)$ & $43.3 \pm 27.5$ & $41.3 \pm 28.8$ & 0.756 \\
\hline $\mathrm{CRP}(\mathrm{mg} / \mathrm{dL})$ & $2.2 \pm 4.4$ & $2.7 \pm 4.5$ & 0.653 \\
\hline Albumin (g/dL) & $3.3 \pm 0.6$ & $3.4 \pm 0.5$ & 0.236 \\
\hline Transthyretin (mg/dL) & $15.5 \pm 6.1$ & $16.8 \pm 5.4$ & 0.300 \\
\hline Total cholesterol $(\mathrm{g} / \mathrm{dL})$ & $162.1 \pm 47.2$ & $170.2 \pm 35.9$ & 0.382 \\
\hline \multicolumn{4}{|c|}{ Cognitive function, physical performance, nutritional status } \\
\hline MMSE (score) & $20.0 \pm 5.6$ & $19.1 \pm 6.8$ & 0.514 \\
\hline Handgrip strength $(\mathrm{kg})$ & $14.7 \pm 5.4$ & $14.1 \pm 6.6$ & 0.644 \\
\hline MSAS (METs) & $2.1 \pm 0.9$ & $1.9 \pm 0.5$ & 0.258 \\
\hline $\mathrm{BI}$ (score) & $39.6 \pm 23.1$ & $35.0 \pm 24.8$ & 0.384 \\
\hline CONUT (score) & $4.6 \pm 2.9$ & $3.5 \pm 2.2$ & 0.061 \\
\hline$\% A C(\%)$ & $97.3 \pm 11.5$ & $97.7 \pm 15.8$ & 0.905 \\
\hline$\%$ TSF $(\%)$ & $72.0 \pm 35.2$ & $73.5 \pm 44.2$ & 0.863 \\
\hline \multicolumn{4}{|l|}{ Swallowing function } \\
\hline FOIS (level) & $5.8 \pm 1.5$ & $4.4 \pm 2.0$ & 0.001 \\
\hline Number of patients with FOIS level $\leq 5$ & $13(31.7)$ & $25(61.0)$ & 0.008 \\
\hline RSST positive & $14(34.2)$ & $24(58.5)$ & 0.026 \\
\hline WST (score) & $4.2 \pm 0.9$ & $3.6 \pm 0.9$ & 0.003 \\
\hline
\end{tabular}

Values are means \pm standard deviation or numbers of subjects per group (n) with percentages.

$\mathrm{AC}$, arm circumference; ACEI, angiotensin converting enzyme inhibitor; $\mathrm{ARB}$, angiotensin receptor blocker; $\mathrm{BI}$, barthel index; $\mathrm{BMI}$, body mass index; CONUT, controlling nutritional status; CRP, C-reactive protein; FOIS, functional oral intake scale; eGFR, estimated glomerular filtration rate; METs, metabolic equivalents; MMSE, mini-mental state examination; MSAS, modified specific activity scale; NT-proBNP, N-terminal pro-B-type natriuretic peptide; NYHA, New York Heart Association; RSST, repetitive saliva swallowing test; TSF, triceps skin fold; WST, water swallowing test. 
Table 7. Outcomes and Rehabilitation Interventions after propensity score matching.

\begin{tabular}{lccr}
\hline & Home $(\mathrm{n}=41)$ & Non-home $(\mathrm{n}=41)$ & P value \\
\hline $\begin{array}{l}\text { Outcomes } \\
\text { Length of hospital stay (day) }\end{array}$ & $29.7 \pm 14.1$ & $45.9 \pm 35.6$ & 0.008 \\
$\begin{array}{l}\text { Discharge disposition } \\
\text { Home }\end{array}$ & $41(100.0)$ & $0(0.0)$ & $<0.001$ \\
$\quad$ Nursing care facility & $0(0.0)$ & $21(51.2)$ & \\
$\quad$ Rehabilitation hospital & $0(0.0)$ & $20(48.8)$ & \\
Rehabilitation interventions & & & \\
PT & $36(87.8)$ & $38(92.7)$ & 0.457 \\
ST & $3(7.3)$ & $9(22.0)$ & 0.061 \\
\hline
\end{tabular}

Values are means \pm standard deviation or numbers of subjects per group (n) with percentages.

PT, physical therapy; ST, swallowing therapy.

multicenter trials and rehabilitation intervention studies are needed to clarify factors that affect discharge disposition in patients with acute exacerbation of HF.

In conclusion, this study indicates that dysphagia hinders patients with acute exacerbation of HF from being discharged to home. Additionally, dysphagia and its severity were independent predictors for discharge to home in patients with acute exacerbation of HF. Thus, early detection and active intervention for dysphagia may be important in improving clinical outcomes in patients with acute exacerbation of HF.

\section{Acknowledgments}

This research received no grant from any funding agency in the public, commercial or not-for-profit sectors. The authors gratefully acknowledge the participation of all study patients and thank all colleagues in our department for their contribution to the medical care of the patients. Also, we would like to thank Editage (https://www.editage.jp) for English language editing.

\section{Conflict of Interest}

The authors declare no conflict of interest.

\section{References}

Adachi, Y., Konishi, H., Fukui, T., Noguchi, T., Kawakami, R., Nakanishi, M., Ibuki, M., Ohara, T., Aihara, N., Takaki, H. \& Goto, Y. (2009) An examination of validity of modified specific activity scale. JJCR, 14, 115-118 (in Japanese).

Belafsky, P.C., Mouadeb, D.A., Rees, C.J., Pryor, J.C., Postma, G.N., Allen, J. \& Leonard, R.J. (2008) Validity and reliability of the Eating Assessment Tool (EAT-10). Ann. Otol. Rhinol. Laryngol., 117, 919-924.

Clark, G.F., Avery-Smith, W., Wold, L.S., Anthony, P. \& Holm, S.E.; Eating, Feeding Task, Force; Commission on Practice (2007) Specialized knowledge and skills in feeding, eating, and swallowing for occupational therapy practice. Am. J. Occup. Ther., 61, 686-700.

Crary, M.A., Mann, G.D. \& Groher, M.E. (2005) Initial psychometric assessment of a functional oral intake scale for dysphagia in stroke patients. Arch. Phys. Med. Rehabil., 86, 1516-1520.

Folstein, M.F., Folstein, S.E. \& McHugh, P.R. (1975) "Minimental state". A practical method for grading the cognitive state of patients for the clinician. J. Psychiatr. Res., 12, 189-198.
Glantz, S.A. \& Slinker, B.K. (2001) Multicollinearity and what to do about it. In Primer of applied regression and analysis of variance, 2nd ed., edited by Glantz, S.A. \& Slinker, B.K. McGraw-Hill Education, New York, NY, pp. 185-240.

Goldman, L., Hashimoto, B., Cook, E.F. \& Loscalzo, A. (1981) Comparative reproducibility and validity of systems for assessing cardiovascular functional class: advantages of a new specific activity scale. Circulation, 64, 1227-1234.

Hansen, T., Kjaersgaard, A. \& Faber, J. (2011) Measuring elderly dysphagic patients' performance in eating: a review. Disabil. Rehabil., 33, 1931-1940.

Hosoya, N., Okada, T. \& Muto, Y. (2001) Japanese anthropometric reference data 2001 (JARD 2001). Jpn. J. Nutr. Assess., 19, 1-81 (in Japanese).

Ignacio de Ulibarri, J., Gonzalez-Madrono, A., de Villar, N.G., Gonzalez, P., Gonzalez, B., Mancha, A., Rodriguez, F. \& Fernandez, G. (2005) CONUT: a tool for controlling nutritional status. First validation in a hospital population. Nutr. Hosp., 20, 38-45.

Isono, N., Imamura, Y., Ohmura, K., Ueda, N., Kawabata, S., Furuse, M. \& Kuroiwa, T. (2017) Transthyretin concentrations in acute stroke patients predict convalescent rehabilitation. J. Stroke Cerebrovasc. Dis., 26, 1375-1382.

Izawa, K., Hirano, Y., Yamada, S., Oka, K., Omiya, K. \& Iijima, S. (2004) Improvement in physiological outcomes and healthrelated quality of life following cardiac rehabilitation in patients with acute myocardial infarction. Circ. J., 68, 315320.

Joundi, R.A., Martino, R., Saposnik, G., Giannakeas, V., Fang, J. \& Kapral, M.K. (2017) Predictors and outcomes of dysphagia screening after acute ischemic stroke. Stroke, 48, 900-906.

Macht, M., King, C.J., Wimbish, T., Clark, B.J., Benson, A.B., Burnham, E.L., Williams, A. \& Moss, M. (2013) Post-extubation dysphagia is associated with longer hospitalization in survivors of critical illness with neurologic impairment. Crit. Care, 17, R119.

Macht, M., Wimbish, T., Clark, B.J., Benson, A.B., Burnham, E.L., Williams, A. \& Moss, M. (2011) Postextubation dysphagia is persistent and associated with poor outcomes in survivors of critical illness. Crit. Care, 15, R231.

Maeda, K. \& Akagi, J. (2015) Decreased tongue pressure is associated with sarcopenia and sarcopenic dysphagia in the elderly. Dysphagia, 30, 80-87.

Mahoney, F.I. \& Barthel, D.W. (1965) Functional evaluation: the barthel index. Md. State Med. J., 14, 61-65.

Morley, J.E. (2018) Editorial: defining undernutrition (malnutrition) in older persons. J. Nutr. Health Aging, 22, 308-310.

Oguchi, K., Saitoh, E., Baba, M., Kusudo, S., Tomomi, T. \& Onogi, K. (2000a) The repetitive saliva swallowing test (RSST) as a screening test of functional dysphagia (2) Validity 
of RSST. Jpn. J. Rehabil. Med., 37, 383-388 (in Japanese).

Oguchi, K., Saitoh, E., Mizuno, M., Baba, M., Okui, M. \& Suzuki, M. (2000b) The repetitive saliva swallowing test (RSST) as a screening test of functional dysphagia (1) normal values of RSST. Jpn. J. Rehabil. Med., 37, 375-382 (in Japanese).

Sansone, G.R., Alba, A. \& Frengley, J.D. (2002) Analysis of FIM instrument scores for patients admitted to an inpatient cardiac rehabilitation program. Arch. Phys. Med. Rehabil., 83, 506-512.

Sasanuma, N., Takahashi, K., Itani, Y., Tanaka, T., Yamauchi, S., Mabuchi, S., Kodama, N., Ishihara, M., Masuyama, T., Miyamoto, Y. \& Domen, K. (2015) Motor and cognitive function analysis for home discharge using the functional independence measure in patients undergoing cardiac rehabilitation at a long-term acute-care hospital. Eur. J. Phys. Rehabil. Med., 51, 781-792.

Serra-Prat, M., Palomera, M., Gomez, C., Sar-Shalom, D., Saiz, A., Montoya, J.G., Navajas, M., Palomera, E. \& Clave, P. (2012) Oropharyngeal dysphagia as a risk factor for malnutrition and lower respiratory tract infection in independently living older persons: a population-based prospective study. Age Ageing, 41, 376-381.

Shimokawa, H., Miura, M., Nochioka, K. \& Sakata, Y. (2015) Heart failure as a general pandemic in Asia. Eur. J. Heart Fail., 17, 884-892.

Tohara, H., Saitoh, E., Mays, K.A., Kuhlemeier, K. \& Palmer, J.B. (2003) Three tests for predicting aspiration without videofluorography. Dysphagia, 18, 126-134.

Tsutsui, H. (2017) Guidelines for diagnosis and treatment of acute and chronic heart failure (JCS 2017 / JHFS 2017). http://www.j-circ.or.jp/guideline/pdf/JCS2017_tsutsui_h.pdf [Accessed: September 21, 2019] (in Japanese).

Yokota, J., Ogawa, Y., Yamanaka, S., Takahashi, Y., Fujita, H., Yamaguchi, N., Onoue, N., Ishizuka, T., Shinozaki, T. \& Kohzuki, M. (2016) Cognitive dysfunction and malnutrition are independent predictor of dysphagia in patients with acute exacerbation of congestive heart failure. PLoS One, 11, e0167326. 